\title{
Transmission of spiking-rate information through layered networks: the role of recurrent and feedback connections Anthony N Burkitt*1,2,3 and Chris Trengove ${ }^{1,3}$
}

\author{
Address: ${ }^{1}$ The Bionic Ear Institute, 384-388 Albert St, East Melbourne, VIC 3002, Australia, ${ }^{2}$ Dept Electrical \& Electronic Engineering, The \\ University of Melbourne, Melbourne, VIC 3010, Australia and ${ }^{3}$ Dept Otolaryngology, The University of Melbourne, Melbourne, VIC 3010 , \\ Australia \\ Email: Anthony N Burkitt* - aburkitt@bionicear.org \\ * Corresponding author
}

from Sixteenth Annual Computational Neuroscience Meeting: CNS*2007

Toronto, Canada. 7-12 July 2007

Published: 6 July 2007

BMC Neuroscience 2007, 8(Suppl 2):P24 doi:10.1 186/I47I-2202-8-S2-P24

(C) 2007 Burkitt and Trengove; licensee BioMed Central Ltd.

\section{Background}

It is a widely accepted tenant that one of the means by which the nervous system and brain encodes information about external stimuli is via the spiking-rate of neurons. Such a spiking-rate code necessitates the transmission of an elevated spiking-rate through successive neurons in a structured network, such as occurs in the auditory and visual pathways. Recent studies, however, have raised serious questions about our understanding of the propagation of such spiking-rate information through structured networks. In networks consisting of successive layers of integrate-and-fire neurons with conductance synapses with feed-forward of information from one layer to the next, it has been found that the mean spiking-rate in deep layers is essentially independent of the input spiking-rate [1]. The neurons within each layer tend to synchronize with each other, resulting in a synchronous volley of action potentials through successive layers, reminiscent of synfire chains. The average spiking-rate in deeper layers either decays to zero or reaches a stable fixed-point, depending upon the model parameters. This behaviour was also observed in an in vitro study using a dynamic patch clamp [2].

\section{Methods}

A network consisting of many layers of neurons is analysed using both analytical and computational techniques. Within each layer the neurons (both excitatory and inhibitory) are recurrently connected. Adjacent layers are connected to each other through both feed-forward and feedback excitatory connections. The neurons receive specific (or driving) excitatory synaptic input from inputs in the previous layer and non-specific (or background) excitatory inputs from neurons outside the layered network. Both Poisson neurons and integrate-and-fire neurons with conductance synapses are analysed [3]. The fixedpoint behaviour of the transmission of spiking-rate information is analysed analytically. Dynamical aspects of the behaviour are analysed computationally.

\section{Results}

It is found that, with a sufficient level of recurrent excitation and background input, the response of the neurons within a layer to input that is external to the layer can be described as a threshold-linear function to a very good approximation over a wide range of input intensities. Activity-dependent synaptic scaling is used to determine the effective gain of the threshold-linear response. The conditions under which spiking-rate information can be reliably transmitted through successive layers are deduced using a fixed-point analysis and are found to depend upon the relative amounts of excitatory feed-forward and feedback input between layers.

\section{Conclusion}

It is found that there is a set of privileged neural parameters allow the propagation of spiking-rate information through deep layered networks and that this set of param- 
eters can arise naturally as a result of simple well-founded principles. This represents a significant result demonstrating that the propagation of spiking-rate information can be achieved when the feedback and recurrent connections, that were absent in previous feed-forward layered models, are incorporated. Also, in contrast to feed-forward models, there is in the present model a clear rationale for the privileged sets of neural parameters that allow the transmission of spiking-rate information.

\section{References}

I. Litvak V, Sompolinsky H, Segev I, Abeles M: On the Transmission of Rate Code in Long Feedforward Networks with Excitatory-Inhibitory Balance. J Neurosci 2003, 23:3006-30I5.

2. Reyes AD: Synchrony-dependent propagation of firing rate in iteratively constructed networks in vitro. Nature Neurosci 2003, 6:593-599.

3. Meffin H, Burkitt AN, Grayden DB: An analytical model for the 'large, fluctuating synaptic conductance state' typical of neocortical neurons in vivo. J Comput Neurosci 2004, 16:159-175.

Publish with Bio Med Central and every scientist can read your work free of charge

"BioMed Central will be the most significant development for disseminating the results of biomedical research in our lifetime. "

Sir Paul Nurse, Cancer Research UK

Your research papers will be:

- available free of charge to the entire biomedical community

- peer reviewed and published immediately upon acceptance

- cited in PubMed and archived on PubMed Central

- yours - you keep the copyright

Submit your manuscript here:

http://www.biomedcentral.com/info/publishing_adv.asp 